\title{
Percepção do estudante sobre a implantação do método OSCE no curso de Odontologia em uma universidade particular
}

\author{
Luís Henrique K. Chaves*, Claudia Tenorio*, Carla Castiglia Gonzaga**, Flares Baratto-Filho**, \\ Rafaela Scariot**, Denise Piotto Leonardi**, Carmen Lucia Mueller Storrer**
}

* Estudante de Pós-Graduação, Escola de Ciências da Vida, Universidade Positivo

** Professor, Escola de Ciências da Vida, Universidade Positivo

Recebido em 05/11/2018. Aprovado em 25/05/2019.

\begin{abstract}
RESUMO
O Objective Structured Clinical Examination (OSCE) é uma avaliação que observa e intervém nas inseguranças dos estudantes em uma condição clínica simulada. Essa forma de avaliar competências, habilidades clínicas, conhecimento, atitudes, comunicação e profissionalismo é considerada uma ferramenta importante no processo de ensino-aprendizagem. Nesse contexto, surgiu a necessidade em implementar, no curso de Odontologia, a avaliação de competência clínica OSCE. O objetivo do estudo foi avaliar a percepção do estudante de Odontologia sobre o método OSCE e seu impacto na formação acadêmica deles. Participaram 34 estudantes do último ano do curso. O instrumento de coleta de dados foi um questionário com opções de respostas ("discordo", "concordo", "concordo parcialmente", "indiferente") que foi respondido voluntariamente. Os dados foram submetidos ao teste Qui-quadrado que comparou as respostas das quatro aplicações da metodologia OSCE durante o ano, com nível de significância de $95 \%(p<0,05)$. Em relação à mesma pergunta, a diferença também foi significativa na última avaliação $(93,1 \% ; p=0,017)$. Quanto ao método ter contribuído para o futuro na prática profissional, 93,1\% $(p=0,042)$ dos estudantes concordaram, na última avaliação do ano. Pode-se concluir, com base na análise de resposta dos questionários nos quatro bimestres, que a percepção dos estudantes de Odontologia do $5^{\circ}$ ano noturno da Universidade Positivo frente à introdução do método OSCE foi bem aceita e positiva, uma vez que a maioria das respostas indicaram que o método contribuiu significativamente para a formação e ofereceu oportunidade de aprendizagem.
\end{abstract}

Descritores: Avaliação Educacional. Odontologia. Educação em Odontologia. OSCE.

\section{INTRODUÇÃO}

Nas últimas décadas um grande interesse pela avaliação de competências e aptidões clínicas e práticas na área da Saúde tem surgido. Dificilmente todas as dimensões e elementos de aprendizagem podem ser avaliadas de forma adequada, por meio das tradicionais formas de avaliação, oral e escrita. Especificamente para o curso de Odontologia, a avaliação do desempenho clínico dos estudantes torna-se essencial, uma vez que realizam procedimentos clínicos nos pacientes desde o primeiro ano do curso de graduação ${ }^{1}$. 
Diferentes métodos de avaliação clínica têm sido propostos na literatura com o intuito de melhorar esse processo, englobando todos os pontos relevantes para a prática clínica e também a fim de torná-lo mais objetivo e consistente. A avaliação formativa é utilizada para melhorar a aprendizagem e, nesse tipo de avaliação, os professores devem se informar sobre o progresso dos estudantes para que se adaptem às necessidades e promovam investimentos nas várias atividades de ensino ${ }^{2,3}$.

A autoavaliação e a discussão com professores e colegas são instrumentos válidos para melhorar a percepção do estudante em relação ao seu próprio desempenho. Essa abordagem pode aumentar a sua autoconfiança e favorecer o aprendizado $^{4}$. Para que os estudantes se autoavaliem, é muito importante que eles tenham o conhecimento da meta desejada e da posição em que estão, além de definir o que é necessário melhorar para alcançá-la ${ }^{5-7}$.

Em 1975, Ronald Harden apresentou uma ferramenta promissora para a avaliação de competências clínicas, aplicada primeiramente em escolas de Medicina, chamada de Objective Structured Clinical Examination (OSCE), definida como uma avaliação do aprendizado cognitivo (conhecimento) e das atitudes/habilidades práticas à partir de um atendimento clínico simulado. Em sua primeira experiência, Harden elaborou uma avaliação com 16 estações, incluindo casos clínicos, atendimento de pacientes e questionários, com dois professores presentes avaliando o atendimento do estudante ${ }^{8,9}$.

O OSCE, atualmente, consiste em situações delimitadas, com roteiros pré-estabelecidos, interação com pacientes simulados, utilizando estações de avaliação em rodízio e que permitem avaliar competências fundamentais, como comunicação e interação com pacientes e familiares; entrevista médica e tomada da história clínica; exame físico geral; raciocínio clínico e formulação de hipóteses; proposição e execução de ações; orientação e educação do paciente. Por esse motivo o OSCE por estações é considerado um dos métodos mais confiáveis para avaliação de competências clínicas de estudantes e residentes, assim como para certificação profissional e avaliação de profissionais médicos em atividade ${ }^{10-}$ 12. A simulação com treinamentos em manequins faz com que a teoria faça sentido na prática, servindo para que o estudante aprimore suas habilidades motoras e confiabilidade diante de um atendimento a pacientes reais nas clínicas ${ }^{13,14}$.

A aplicação do OSCE apresenta grande parte dos critérios de uma boa avaliação, como validade (afere o grau no qual o teste realmente avalia aquilo que propõe), confiabilidade (afere a reprodutibilidade e a consistência de um teste) e viabilidade (promoção de resultados e feedback, produzindo melhor suporte educacional) $)^{17,18}$. Além de avaliar a competência clínica dos estudantes, o método OSCE permite a autoavaliação por parte dos alunos, assim como um momento de devolutiva dos resultados e discussão com o professor. Essa autoavaliação e feedback são instrumentos válidos para melhorar a percepção do estudante no que se refere ao seu próprio desempenho. Esses critérios avaliativos já foram analisados com excelentes resultados ${ }^{19,20}$.

Nesse contexto, surgiu a necessidade em implementar, no curso de Odontologia da Universidade Positivo, a avaliação de competência clínica pelo método OSCE. Sendo assim, o objetivo deste estudo foi avaliar a percepção dos estudantes de Odontologia do último ano sobre o método OSCE, por meio de um questionário estruturado, aplicado após cada uma das quatro avaliações bimestrais.

\section{METODOLOGIA}

O estudo foi aprovado pelo Comitê de Ética em Pesquisa institucional (CAAE 69486617. $5.0000 .0093)$

Participaram da pesquisa 34 estudantes que 
estavam cursando o $5^{\circ}$ ano noturno do curso. $\mathrm{O}$ instrumento para a coleta de dados foi um questionário estruturado com 7 questões sobre a percepção do estudante em relação à aplicação da metodologia OSCE.

Antes do início da aplicação do método, os estudantes participantes permaneceram confinados em uma sala e receberam orientações de como seria o fluxo, tempo e avaliação entre as estações. Os atendimentos simulados com atores foram realizados em 5 minutos, tempo para que os estudantes analisassem a questão e desenvolvessem a resolução para o caso apresentado. Após esses atendimentos simulados, seguiam para as estações seguintes, com mais 5 minutos para analisar e resolver cada um dos problemas. Essas tarefas poderiam contemplar respostas escritas, práticas ou orais que variaram ao longo do ano em diferentes áreas do conhecimento da Odontologia, como Endodontia, Estomatologia, Periodontia, Anatomia/Cirurgia, Prótese, Radiologia e Dentística. Ao final do circuito das estações, os estudantes responderam ao questionário estruturado, relativo à sua percepção sobre a metodologia. Para cada uma das perguntas, tinha como opções de respostas ("discordo", "concordo", "concordo parcialmente" e "indiferente") (tabela 1).

Tabela 1. Perguntas contidas nos questionários sobre a percepção dos alunos em relação ao método OSCE

\begin{tabular}{cl} 
Ordem das questões & \multicolumn{1}{c}{ Perguntas sobre a Metodologia aplicada } \\
\hline 1 & O método OSCE contribui para seu futuro na prática profissional? \\
2 & O método OSCE avalia as competências clínicas odontológicas adequadamente? \\
3 & Esse método contribui para você analisar suas limitações na prática odontológica? \\
4 & O cenário e encenação foram satisfatórios? \\
5 & Você acha que esse método deve ser implementado no curso de Odontologia? \\
6 & O método foi eficiente e lhe ofereceu oportunidade de aprendizagem? \\
7 & O método contribuiu para a sua formação?
\end{tabular}

Foram realizadas 4 aplicações da metodologia OSCE ao longo do ano letivo, divididas por bimestres (primeiro ao quarto bimestre). Os professores avaliadores possuíam uma ficha para observação de desempenho, com perguntas que deveriam fazer para o diagnóstico do caso, além de atenção e educação para com o paciente. Todos os atendimentos foram filmados e analisados. Os três melhores atendimentos foram reproduzidos em sala de aula, com o consentimento dos estudantes, no dia da devolutiva dos atendimentos.

Para comparar as respostas das 4 aplicações da metodologia OSCE durante o ano, os dados foram submetidos ao teste de qui-quadrado, com nível de significância de 5\%. Todas as análises foram realizadas com o software Statistical Package for Social Science (SPSS; version 20.0; SPSS Inc. Chicago, IL, USA).

\section{RESULTADOS}

Os resultados das comparações das respostas dos estudantes em relação à percepção da metodologia OSCE do primeiro ao quarto bimestre estão demonstrados em gráficos (figura 1). Quando comparadas as respostas dos estudantes em relação ao método, não houve diferença estatística para as questões entre os bimestres $(p>0,05)$. Os estudantes não tiveram uma percepção diferente entre o primeiro, segundo e terceiro bimestre. 
Q1: O método OSCE contribuiu para o seu futuro na prática profissional?

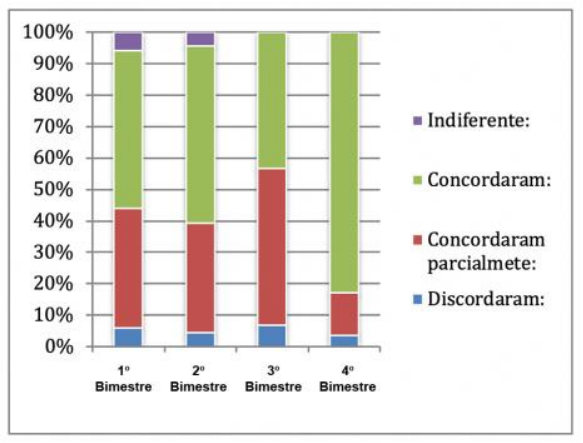

Q3: Esse método contribui para você analisar suas limitações na prática odontológica?

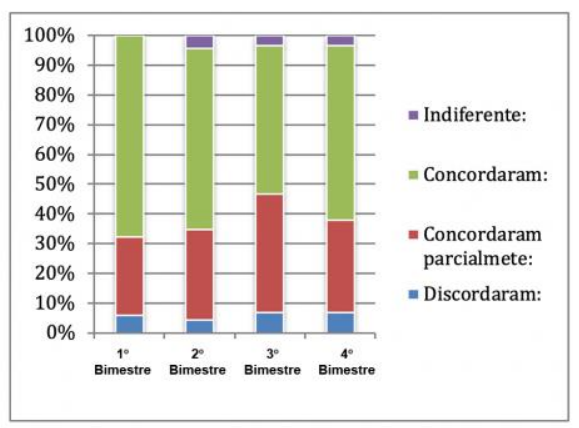

Q5: Você acha que esse método deve ser implementado no curso de Odontologia?

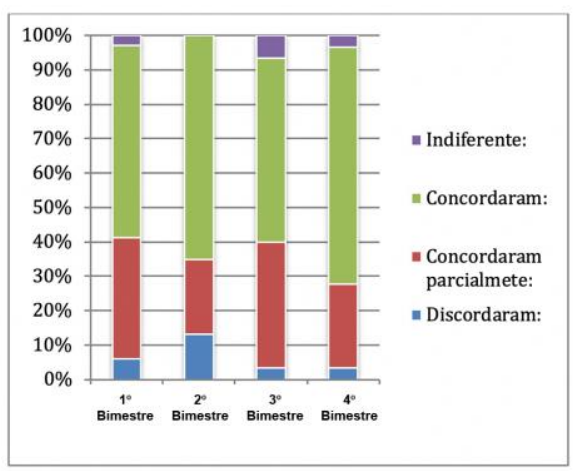

Q2: O método OSCE avalia as competências clínicas odontológicas adequadamente?

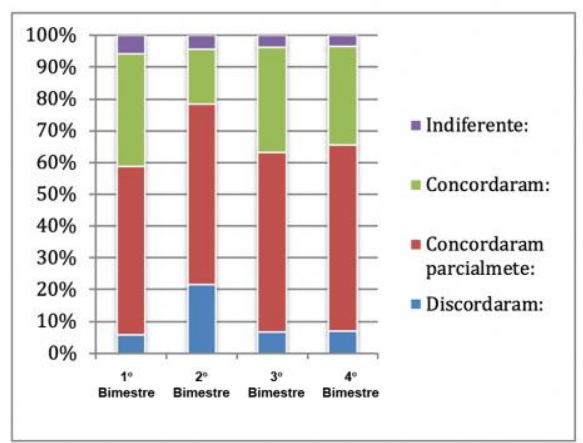

Q4: O cenário e encenação foram satisfatórios?

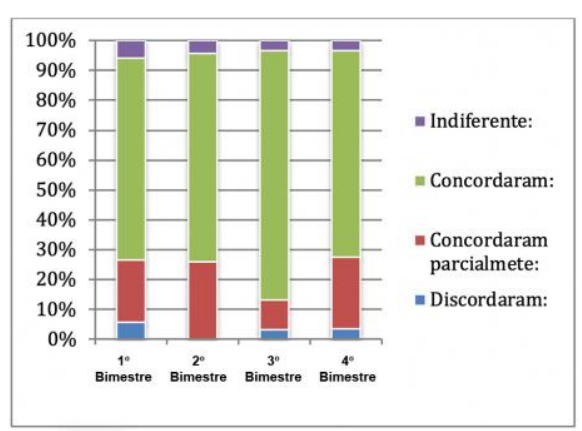

Q6: O método foi eficiente e lhe ofereceu oportunidade de aprendizagem?

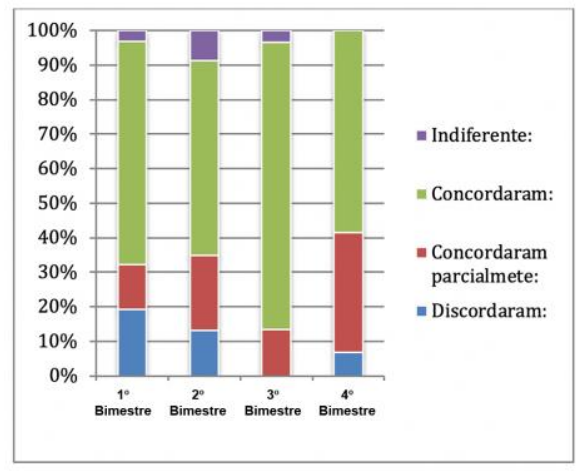

Q6: O método foi eficiente e lhe ofereceu oportunidade de aprendizagem?

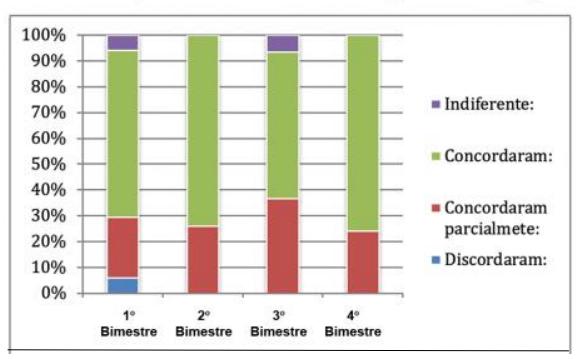

Figura 1. Relação das frequências de respostas em porcentagem dos alunos a cada pergunta (Q1 a Q7) do questionário avaliativo da metodologia OSCE nos 4 bimestres 
A tabela 2 mostra as frequências absolutas e relativas na comparação entre as respostas para o primeiro e o quarto bimestres, em que são observadas diferenças estatisticamente significantes para as questões Q1 e Q6 ( $\mathrm{p}=0,042$ e $\mathrm{p}=0,017$, respectivamente). Esses resultados mostram melhora na percepção dos estudantes no quarto bimestre, em relação à primeira avaliação.

Tabela 2. Comparação das respostas dos alunos em relação as perguntas dos questionários (Q1 a Q7) com diferença estatística nas aplicações do OSCE. Os valores mostrados se referem às frequências absolutas e relativas

\begin{tabular}{|c|c|c|c|c|c|c|c|c|}
\hline & & Q1 & Q2 & Q3 & Q4 & Q5 & Q6 & Q7 \\
\hline & & n $(\%)$ & n (\%) & n $(\%)$ & n $(\%)$ & n $(\%)$ & n $(\%)$ & n $(\%)$ \\
\hline \multirow{4}{*}{$\begin{array}{l}\text { Primeiro } \\
\text { bimestre }\end{array}$} & Indiferente & $2(5,9)$ & $2(5,9)$ & $0(0,0)$ & $2(5,9)$ & $1(2,9)$ & $4(11,8)$ & $2(5,9)$ \\
\hline & Discordo & $2(5,9)$ & $2(5,9)$ & $2(5,9)$ & $2(5,9)$ & $2(5,9)$ & $6(17,6)$ & $2(5,9)$ \\
\hline & $\begin{array}{c}\text { Concordo } \\
\text { parcialmente }\end{array}$ & $13(38,2)$ & $18(52,9)$ & $9(26,5)$ & $7(20,6)$ & $12(35,3)$ & $4(11,8)$ & $9(26,5)$ \\
\hline & Concordo & $17(50,0)$ & $12(35,3)$ & $23(67,6)$ & $23(67,6)$ & $19(55,9)$ & $20(58,8)$ & $21(61,8)$ \\
\hline \multirow{5}{*}{$\begin{array}{l}\text { Quarto } \\
\text { bimestre }\end{array}$} & Indiferente & $1(3,4)$ & $1(3,4)$ & $1(3,4)$ & $1(3,4)$ & $1(3,4)$ & $0(0,0)$ & $0(0,0)$ \\
\hline & Discordo & $1(3,4)$ & $2(6,9)$ & $2(6,9)$ & $1(3,4)$ & $1(3,4)$ & $2(6,9)$ & $0(0,0)$ \\
\hline & $\begin{array}{c}\text { Concordo } \\
\text { parcialmente }\end{array}$ & $3(10,3)$ & $17(58,6)$ & $9(31,0)$ & $7(24,1)$ & $7(24,1)$ & $10(34,5)$ & $9(31,0)$ \\
\hline & Concordo & $24(82,8)$ & $9(31,0)$ & $17(58,6)$ & $20(69,0)$ & $20(69,0)$ & $17(58,6)$ & $20(69,0)$ \\
\hline & Valor-p & $0,042 *$ & 0,940 & 0,595 & 0,921 & 0,729 & $0,017 *$ & 0,160 \\
\hline
\end{tabular}

*: teste do qui-quadrado, com nível de significância de 0.05

\section{DISCUSSÃO}

O OSCE, como método de avaliação clínico e pré-clínico, apresenta algumas vantagens como uma avaliação mais objetiva, seleção de tarefas e competências a serem avaliadas, mas também a possibilidade de avaliar habilidades de comunicação estudante/paciente, profissionalismo, manejo do tempo, pensamento crítico, relações interpessoais e ética profissional.
Os resultados do presente estudo mostraram que mais de metade dos estudantes ao longo dos 4 bimestres $(62,86 \%$ em média) concordaram de que o método deveria ser implementado no curso de Odontologia, sugerindo de que a sua percepção foi positiva em relação à implantação do método.

As habilidades de comunicação puderam ser observadas pelos professores avaliadores na 
realização das estações simuladas, nas quais os estudantes interagiram com um paciente/ator para obter informações para o diagnóstico da doença. No entanto, existem algumas desvantagens em relação ao método, que também foram observadas no presente estudo. Dentre elas estão o tempo reduzido de permanência nas estações, a impossibilidade de simular procedimentos invasivos em pacientes reais, ansiedade dos estudantes, dificuldade de mobilização de um grande número de examinadores e pacientes/atores, assim como custo mais elevado em comparação aos outros métodos de avaliação ${ }^{17-19}$.

No presente estudo foi observada uma dificuldade e resistência inicial dos estudantes frente a uma nova forma de avaliação. Este fato pode ser observado na pergunta Q6, quando comparados os dados do primeiro e do quarto bimestre, mostrando um crescimento nas respostas positivas, mostrando que o método foi eficaz e ofereceu oportunidade de aprendizagem. Essa mudança significativa da avaliação inicial para a final pode ser entendida como uma familiarização dos estudantes com o método ao longo do tempo, além de mostrar um aumento na aceitação em relação ao primeiro bimestre, momento do primeiro contato com a metodologia.

Apesar de não ter sido realizada nenhuma avaliação sobre a ansiedade e nervosismo dos estudantes, esses comportamentos foram frequentemente observados, principalmente nas primeiras aplicações do OSCE, o que pode ser explicado pelo ambiente da própria avaliação e por ser a primeira vez que eles estavam entrando em contato com esse tipo de exame. No entanto, no quarto bimestre os estudantes tiveram maior segurança e aceitação do método, fato que também é refletido pelo maior número de respostas positivas na questão $\mathrm{Q} 1$, que avaliava se a metodologia contribui para o futuro na prática profissional. No primeiro bimestre apenas metade dos estudantes concordavam (50\%), já no quarto bimestre houve um aumento significativo no número de alunos que concordaram com essa pergunta $(82,8 \%)$. Os relatos dos estudantes após o término dessa experiência foram de que o OSCE é uma avaliação mais objetiva e aproximada da realidade de atividade clínica, concordando com a literatura ${ }^{18-21}$.

A etapa final do OSCE é o feedback (devolutiva), que tem como objetivo observar o desempenho do estudante na estação, gerando informações para comparação entre o aprendizado demonstrado e o esperado. Durante esta etapa, é fundamental discutir essas informações para que o estudante possa confirmar, adicionar, substituir, ajustar ou reestruturar o conhecimento na memória ${ }^{17,20}$. No presente estudo, a devolutiva foi realizada uma semana após a aplicação do método OSCE. Neste momento os professores apontaram como deveria ser realizada a tarefa proposta e os principais erros cometidos. Também foram mostrados os três melhores atendimentos, com o consentimento dos responsáveis. O importante é que o estudante conheça de forma breve como foi o seu desempenho para reforçar as respostas corretas, superar suas deficiências e corrigir erros cometidos. Os professores devem identificar essas deficiências para a correção, antes que o estudante ingresse nas disciplinas clínicas do curso de Odontologia. Os professores de cada área também devem se reunir para analisar erros mais recorrentes durante a avaliação e apontar estratégias e medidas para reforçar o ensino.

O OSCE pode ser considerado como uma maneira para avaliar as competências odontológicas, porém as dificuldades na logística da sua implementação fazem que a sua aplicação possa ser limitada, por isso a avaliação deve ser muito bem organizada antes do seu início, com o número de professores e ajudantes previamente 
estabelecido.

Finalmente, deve-se considerar que a aplicação contínua e sistemática do OSCE pode impactar favoravelmente na formação dos futuros cirurgiões-dentistas, considerando que este método, provavelmente, é o que melhor demonstra que a avaliação é uma ferramenta importante e fundamental no processo formativo $^{13,22}$. Apesar do desafio, a experiência foi aplicada com êxito, permitindo identificar pontos fortes e deficiências dos alunos participantes.

\section{CONCLUSÃO}

Pode-se concluir, baseado na análise de resposta dos questionários nos quatro bimestres, que a percepção dos estudantes de Odontologia do $5^{\circ}$ ano noturno da Universidade Positivo frente à introdução do método OSCE foi bem aceita e positiva, uma vez que a maioria das respostas indicaram que o método contribuiu significativamente para a formação e ofereceu oportunidade de aprendizagem.

\section{ABSTRACT \\ Student's perception of the implementation of the OSCE method in the Dentistry course of a private university}

The Objective Structured Clinical Examination (OSCE) is used to detect and intervene in the students' lack of confidence in a simulated clinical condition. The OSCE assesses competences, clinical skills, knowledge, attitude, communication, and professionalism, being considered an important tool in the teachinglearning process. This context raised the need to implement the OSCE clinical competence assessment. The objective of the study was to assess the dentistry student's perception of the OSCE method and its impact on their academic training. This study included thirty-four senior students. The data were collected using a multiple-choice questionnaire ("disagree", "agree", "partially agree", "indifferent") voluntarily answered. The chi-square test was used to analyze the data by comparing the answers of four OSCE methodology applications during the year, with a 95\% (p <0.05) significance level. The difference was also significant regarding the same question in the last assessment $(93.1 \%, p=0.017)$. As for the method having contributed to their future professional practice, $93.1 \%(p=0.042)$ of the students agreed in the last assessment of the year. Based on the analysis of questionnaire answers in the four bimesters, we concluded that the perception of the students attending the $5^{\text {th }}$ year of dentistry at Positivo University is that the implementation of the OSCE method was well accepted and positive, since most of the answers indicated that the method significantly contributed to their training and provided learning opportunities.

Descriptors: Educational Assessment. Dentistry. Education in Dentistry. OSCE.

\section{REFERÊECIAS}

1. Nie R, Zhu F, Meng X, Zhang H, Xie S, Wu L, Yang J, et al. Application of OSCE for Stage Assessment in Standardized Training for Oral Residents. J Dent Educ. 2018;82(9):1000-6.

2. Steiner-Oliveira C, Kantovitz KR, Teixeira MS, Vieira KA, Serra MD, Gambarelli, FR et al. Estudo preliminar do instrumento de avaliação clínica proposto aos alunos de graduação de Odontopediatria da Faculdade de Odontologia de Piracicaba - Unicamp. Ciênc Odontol Bras. 2006;9(1):77-86.

3. Logar GA, Coelho COL, Pizi ECG, Galhano GAP, Neves AP, de Oliveira LT, Bertão JMO. OSCE na avaliação clínica odontológica: relato de experiência com estudantes de graduação. Rev ABENO. 2018;18(1):15-24.

4. Amaral FTV, Troncon, LEA. Participação de estudantes de Medicina como avaliadores em Exame Estruturado de Habilidade Clínicas (OSCE). Rev Bras Educ Med. 2017;31(1):81-9.

5. Deci EL, Ryan RM. Promoting self- 
determined education. Scand J Educ Res. 1994;38(1):3-14.

6. Kupke J, Wicht MJ, Stützer H, Derman SH, Lichtenstein NV, Noack MJ. Does the use of a visualized decision board by undergraduate students during shared decision-making enhance patients' knowledge and satisfaction? A randomised controlled trial. Eur J Dent Educ. 2013;17(1):19-25.

7. Manogue, M, Brown G, Foster H. Clinical assessment of dental students: values and practices of teachers in restorative dentistry. Med Educ. 2001;35(4):364- 70.

8. Mania TV, Vargas AMD, Ferreira EF. Inserção no mundo do trabalho odontológico: percepção de graduandos em odontologia sobre habilidades adquiridas e expectativas. Rev ABENO. 2018;18(3):14858.

9. Gonçalves PVAJ, Pretti H, Teixeira KIR, Magalhães CS, Moreira NA, Peixoto RTRC. Estratégias para avaliação do desempenho clínico de estudantes de Odontologia. Rev Docência Ens Sup. 2016;6(2):223-46.

10. Harden RM, Gleeson FA. Assessment of clinical competence using an objective structured clinical examination (OSCE). Med Educ. 1979;13(1):41-54.

11. Harden RM. What is an OSCE? Med Teach.1988;10(1):19-22.

12. Harden, RM. Twelve tips for organizing an Objective Structured Clinical Examination (OSCE). Med Teach. 1990;12(3-4):259-64.

13. Lele SM. A mini-OSCE for formative assessment of diagnostic and radiographic skills at a Dental College in India. J Dent Educ. 2011;75(12):1583-9.

14. Park SE, Kim A, Kristiansen J, Karimbux NY. The influence of examiner type on Dental students OSCE scores. J Dent Educ. 2015;79(1):89-94.
15. Macluskey M, Durham J, Balmer C, Bell A, Cowpe J, Dawson L, et al. Dental student suturing skills: a multicentre trial of a checklist-based assessment. Eur J Dent Educ. 2011;15(4):244-9.

16. Landes C, Höfer SH, Shuebel F, Ballon A, Teiler A, Tran A,Weber A, et al. Long- term, prospective teaching effectivity of practical skills. J Craniomaxillofac Surg. 2014;42(5):97-104.

17. Barman, A. Critiques on the objective structured clinical examination. Ann Acad Med Singapore. 2005;34(8):478-82.

18. Graham R, Zubiaurre Bitzer LA, Mensah FM, Anderson OR. Dental student perceptions of the educational value of a comprehensive, multidisciplinary OSCE. J Dent Educ. 2014;78(5):694-702.

19. Graham R, Zubiaurre Bitzer LA, Anderson OR. Reliability and predictive validity of a comprehensive preclinical OSCE in dental education. J Dent Educ.2013;77(2):161-7.

20. Eberhard L, Hassel A, Baumer A, Becker F, Beck-Mubotter J, Bomicke W, et al. Analysis of quality and feasibility of an objective stuctured clinical examination. Eur J Dent Educ. 2011;15(3):172-8.

21. Mossey PA, Newton JP, Stirrups DR. Scope of the OSCE in the assessment of clinical skills in dentistry. $\mathrm{Br}$ Dent $\mathrm{J}$. 2001;190(6):323-6.

22. Höfer SH, Schuebel F, Sader R, Landes C. Development and implementation of an objective structured clinical examination (OSCE) in CMF surgery for dental students. J Craniomaxillofac Surg. 2013;41(5):412-6.

\section{Correspondência para:}

Carmen L. Mueller Storrer

e-mail: carmen.storrer@gmail.com

R. Prof. Pedro Viriato Parigot de Souza, 5300 -

Cidade Industrial

81280-330 Curitiba/PR 